\title{
Migalastat improves diarrhea in patients with Fabry disease: clinical-biomarker correlations from the phase 3 FACETS trial
}

\author{
Raphael Schiffmann ${ }^{1,14^{*}}$, Daniel G. Bichet ${ }^{2}$, Ana Jovanovic ${ }^{3}$, Derralynn A. Hughes ${ }^{4}$, Roberto Giugliani ${ }^{5}$, \\ Ulla Feldt-Rasmussen ${ }^{6}$, Suma P. Shankar ${ }^{7,13}$, Laura Barisoni ${ }^{8}$, Robert B. Colvin ${ }^{9}$, J. Charles Jennette ${ }^{10}$, \\ Fred Holdbrook ${ }^{11}$, Andrew Mulberg ${ }^{11}$, Jeffrey P. Castelli ${ }^{11}$, Nina Skuban ${ }^{11}$, Jay A. Barth ${ }^{11}$ and Kathleen Nicholls ${ }^{12}$
}

\begin{abstract}
Background: Fabry disease is frequently characterized by gastrointestinal symptoms, including diarrhea. Migalastat is an orally-administered small molecule approved to treat the symptoms of Fabry disease in patients with amenable mutations.

Methods: We evaluated minimal clinically important differences (MCID) in diarrhea based on the corresponding domain of the patient-reported Gastrointestinal Symptom Rating Scale (GSRS) in patients with Fabry disease and amenable mutations $(N=50)$ treated with migalastat $150 \mathrm{mg}$ every other day or placebo during the phase 3 FACETS trial (NCT00925301).
\end{abstract}

Results: After 6 months, significantly more patients receiving migalastat versus placebo experienced improvement in diarrhea based on a MCID of 0.33 (43\% vs 11\%; $p=.02$ ), including the subset with baseline diarrhea (71\% vs 20\%; $p=.02$ ). A decline in kidney peritubular capillary globotriaosylceramide inclusions correlated with diarrhea improvement; patients with a reduction > 0.1 were 5.6 times more likely to have an improvement in diarrhea than those without $(p=.031)$.

Conclusions: Migalastat was associated with a clinically meaningful improvement in diarrhea in patients with Fabry disease and amenable mutations. Reductions in kidney globotriaosylceramide may be a useful surrogate endpoint to predict clinical benefit with migalastat in patients with Fabry disease.

Trial registration: NCT00925301; June 19, 2009.

Keywords: Amenable mutation, Diarrhea, Fabry disease, Gastrointestinal, Globotriaosylceramide, GSRS, Lyso-Gb ${ }_{3}$, Migalastat, Pharmacological chaperone

\section{Background}

Fabry disease is a rare, progressive, life-threatening Xlinked lysosomal storage disorder, affecting males and females, with an estimated prevalence of $1: 117,000$ to 1 : $40,000[1,2]$. Mutations in the GLA gene can lead to a deficiency of the lysosomal enzyme $\alpha$-galactosidase A, which in turn results in an accumulation of glycosphingolipids, including globotriaosylceramide (GL-3) and plasma globotriaosylsphingosine (lyso- $\mathrm{Gb}_{3}$ ), and subsequently the

\footnotetext{
* Correspondence: raphael.schiffmann@BSWhealth.org

${ }^{1}$ Baylor Scott \& White Research Institute, Dallas, TX, USA

${ }^{14}$ Institute of Metabolic Disease, 3812 Elm Street, Dallas, TX 75226, USA

Full list of author information is available at the end of the article
}

debilitating signs, symptoms, and life-limiting sequelae of Fabry disease [3]. Intrafamilial phenotypic variability is common in Fabry disease [4] and other genetic disorders, such as muscular dystrophy [5], making it difficult to provide an accurate prognosis to patients based only on family history. Levels of disease substrate have been used as biomarkers in various clinical studies in Fabry disease $[6,7]$; however, the correlation of changes in these biomarkers with clinical variables remains limited.

Gastrointestinal signs and symptoms are a prominent and clinically important manifestation of Fabry disease and are reported by at least half of patients [8, 9]. Common gastrointestinal signs and symptoms associated

(C) The Author(s). 2018 Open Access This article is distributed under the terms of the Creative Commons Attribution 4.0 International License (http://creativecommons.org/licenses/by/4.0/), which permits unrestricted use, distribution, and 
with Fabry disease include diarrhea, nausea, vomiting, abdominal pain, and constipation [10, 11]. Gastrointestinal manifestations of Fabry disease are reported from an early age, and often have profound negative effects on social and economic functioning and quality of life in patients $[11,12]$.

Migalastat is a pharmacological chaperone designed to bind selectively and reversibly with high affinity to the active sites of certain mutant forms of $\alpha$-galactosidase (amenable GLA mutations) [13, 14]. Chaperoning mutated $\alpha$-galactosidase A to lysosomes may mimic natural enzyme trafficking, which has been suggested to result in more consistent $\alpha$-galactosidase A activity than current standard of care enzyme replacement therapy (ERT) [15].

In the phase 3 FACETS trial, which included a 6month placebo-controlled stage, treatment with migalastat maintained stable renal function, reduced cardiac mass, and reduced the severity of gastrointestinal signs and symptoms (diarrhea, reflux, and indigestion domains) in patients with Fabry disease and amenable mutations [15]. In the phase 3, active-controlled ATTRACT study, migalastat and ERT had similar effects on renal function in patients with Fabry disease and amenable mutations, and cardiac mass decreased significantly with migalastat treatment (compared with no change with ERT); furthermore, migalastat was generally safe and well-tolerated [16]. These results led to the approval of migalastat in the European Union, Switzerland, Canada, Australia, Republic of Korea, Japan, and Israel for the treatment of Fabry disease in patients aged 16 years and older with amenable mutations $[14,17]$.

We report here the results of further analyses using minimal clinically important difference (MCID) to evaluate improvements in diarrhea using the patient-reported Gastrointestinal Symptom Rating Scale (GSRS), in patients with Fabry disease treated with migalastat in the FACETS study. We also examine whether reductions in kidney peritubular capillary (PTC) GL-3 or lyso-Gb ${ }_{3}$ can be used as a surrogate endpoint to predict clinical benefit with migalastat.

\section{Methods}

\section{Study design and patients}

The FACETS trial (AT1001-011, NCT00925301) has been described previously [15]. In brief, the study consisted of a 6-month randomized, double-blind, placebo-controlled phase, followed by a 6-month open-label phase with cross-over of placebo-treated patients to migalastat, and a 12-month extension phase. Male and female patients aged 16 to 74 years with Fabry disease, who were naive to ERT or had not received ERT for at least 6 months before screening, were eligible for randomization [15]. The effect of migalastat on gastrointestinal symptoms was evaluated in patients with GLA mutations amenable to migalastat $(N=50)$ [13].

\section{Gastrointestinal symptoms rating scale}

The GSRS comprises 15 questions that assess the severity of 5 domains: diarrhea ("GSRS-D"), abdominal pain, constipation, indigestion, and reflux. Each domain consists of 2-4 questions, each rated on a 7-point Likert scale (from 1-absence of burden to 7-very severe discomfort) [18]. The GSRS-D has 3 questions to assess diarrhea frequency, consistency, and urgency; scores were determined by calculating the mean of the items within this domain. Results were collected at baseline and months $6,12,18$, and 24 for all patients with amenable mutations, and for the subset of patients presenting with gastrointestinal signs and symptoms at baseline.

\section{GL-3 levels in kidney peritubular capillaries}

Detailed methodology and results of the qualitative assessments of kidney biopsies have been reported $[15,19]$. Briefly, kidney biopsies were performed at baseline and at months 6 and 12; these were assessed by 3 independent pathologists using whole slide images at $100 \times$ magnification in at least 300 peritubular capillaries in each biopsy to quantify the average number of GL-3 inclusions per PTC. Response to treatment was defined as a reduction of $>0.1$ inclusions per capillary (which is above the level of background staining).

\section{Plasma lyso-Gb ${ }_{3}$}

Plasma lyso- $-\mathrm{Gb}_{3}$ levels were assessed at baseline and at months 6 and 12, and analyzed by means of liquid chromatography-mass spectroscopy [15]. The liquid chromatography-mass spectroscopy plasma lyso-Gb method used a novel stable isotope-labeled internal standard, ${ }^{13} \mathrm{C}_{6}$-lyso- $\mathrm{Gb}_{3}$ (lower-limit-of-quantification: 0 . $200 \mathrm{ng} / \mathrm{mL}, \quad 0.254 \mathrm{nmol} / \mathrm{L}) \quad[20,21]$. Response to treatment was defined as any reduction from baseline.

\section{Statistical analyses}

The mean change in GSRS scores from baseline to month 6 was a pre-specified endpoint in the FACETS study. Change from baseline was presented descriptively; statistical tests of significance were performed using an ANCOVA model that included treatment, baseline, and treatment-by-baseline interaction. The $p$-value was calculated based on the comparison of the least squares means.

A response in the GSRS-D was defined as a reduction of 0.33 from baseline (i.e., MCID). The MCID was based on estimates in the literature for several non-Fabry gastrointestinal disorders in which diarrhea is a prominent symptom, and is consistent with an estimate of MCID based on data in Fabry patients from the FACETS study. Specifically, the MCID of 0.33 was derived from 
anchor-based methodologies from liver transplant patients with gastrointestinal symptoms $(\mathrm{MCID}=0.33)$ [22], patients with autoimmune disease with and without gastrointestinal symptoms $(\mathrm{MCID}=0.33)$ [23], and renal transplant patients with and without gastrointestinal symptoms $(\mathrm{MCID}=0.40)$ [24]. A distribution-based estimate of MCID in Fabry disease was derived from the change from baseline data in the placebo arm of the FACETS study. Using this approach, an MCID of 0.35 was generated, based on half the standard deviation [22, 23], supporting an MCID for GSRS-D of 0.33 in Fabry patients. A sensitivity analysis using a higher threshold of 0 . 66 was also performed to confirm the results.

The number of patients demonstrating a response in GSRS-D and/or PTC GL-3 from baseline to month 6 was compared between treatment groups. A retrospective analysis using Xu's statistic, a multivariate test used to assess if treatment has a beneficial effect on multiple outcomes simultaneously [25], evaluated whether treatment impacted both parameters as a combined endpoint. Logistic regression was used to assess the correlation between changes in GSRS-D and PTC GL-3. A similar regression analysis was performed to assess the correlation between changes in GSRS-D and plasma lyso- $\mathrm{Gb}_{3}$.

Pre-specified analyses were conducted for all patients with amenable mutations and, post hoc, for the subset of patients with amenable mutations who reported diarrhea symptoms at baseline.

\section{Results}

\section{Summary of GSRS findings}

Of the 50 patients with Fabry disease and amenable mutations who were enrolled in the FACETS study, 28 (56\%) reported diarrhea symptoms at baseline.

As previously reported [15], in patients randomly assigned to migalastat $(n=28)$, symptoms of diarrhea, based on the GSRS-D, improved within the first 6 months of treatment (change from baseline, -0.3 ), whereas diarrhea in the placebo-treated group $(n=22)$ worsened (change from baseline, $+0.2 ; p=.03$ ). A numerically larger reduction in GSRS-D scores was also observed in the subgroup of patients who reported gastrointestinal symptoms at baseline (migalastat change from baseline, -0.6 ; placebo change from baseline, +0.2 ). These improvements continued through 24 months of treatment [15].

\section{Minimal clinically important difference analysis}

After 6 months of treatment, 12/28 (43\%) migalastattreated patients experienced a GSRS-D score improvement of 0.33 (i.e., MCID) compared with $2 / 19$ (11\%) patients receiving placebo $(p=.02)$ (Fig. 1a, b). In the subset of patients with diarrhea symptoms at baseline (baseline GSRS-D scores $\geq 1$ ), 12/17 (71\%) of the migalastat-treated patients experienced a clinically relevant improvement of 0.33 compared with 2/10 (20\%) of placebo-treated patients $(p=.02)$ (Fig. 1a, b).

\section{Sensitivity analyses}

The results of the MCID analysis for observed improvement in diarrhea were confirmed in a sensitivity analysis. Using an improvement threshold of 0.66, 9/ 28 (32\%) migalastat-treated patients experienced a clinically relevant change compared with $1 / 19$ (5\%) placebo-treated patients $(p=.03)$. In patients with diarrhea symptoms at baseline (baseline GSRS-D score of $\geq 1$ ), 9/13 (69\%) migalastat-treated patients experienced a clinically relevant change compared with $1 / 9(11 \%)$ placebo-treated patients $(p=.01)$ (Fig. 1c).

\section{GSRS-D and kidney GL-3 inclusions}

As previously reported, 6 months of migalastat treatment was associated with a significantly greater reduction in the mean number of GL-3 inclusions per PTC compared with placebo $(-0.25$ vs +0.07 ; $p=.008$ ) [15]. An analysis conducted on a combined endpoint of mean change from baseline in PTC GL-3 inclusions and GSRS-D using Xu's statistic demonstrated a significant treatment effect of migalastat versus placebo (1-sided; $p=.009$ ).

Assessment of patient-level responses demonstrated a consistent beneficial effect of migalastat on PTC GL-3 inclusions and GSRS-D. A majority of patients with amenable mutations $(15 / 18 ; 83 \%)$ treated with migalastat demonstrated a response in PTC GL-3 and/or GSRS$D$ when either or both of these endpoints were elevated at baseline, compared with 5/15 (33\%) patients treated with placebo.

A logistic regression modeling the improvement in GSRS-D (ie, change from baseline to Month $6<-0$. 33) as the dependent variable, and reduction in PTC GL-3 inclusions (ie, change from baseline $<-0.1, \geq-0.1$ ) and treatment group as independent variables, indicated that reductions in PTC GL-3 inclusions were strongly associated with improvement in diarrhea (Table 1). Patients who had a reduction of $>0.1$ in PTC GL-3 inclusions (ie, change from baseline <-0.1) were 5.6 times more likely to have an improvement in diarrhea symptoms than patients who did not have a reduction $(p=.031)$.

\section{GSRS-D and plasma lyso- $\mathrm{Gb}_{3}$}

When the same correlation analysis was performed for changes in plasma lyso- $\mathrm{Gb}_{3}$, defined as any reduction from baseline, and a response in GSRS-D, defined as any reduction $\geq 0.33$, the linear correlation was not statistically significant (odds ratio $=2.5$; $95 \%$ confidence interval $0.63-9.6 ; p=.2$ ). When the logistic regression was conducted excluding patients with 

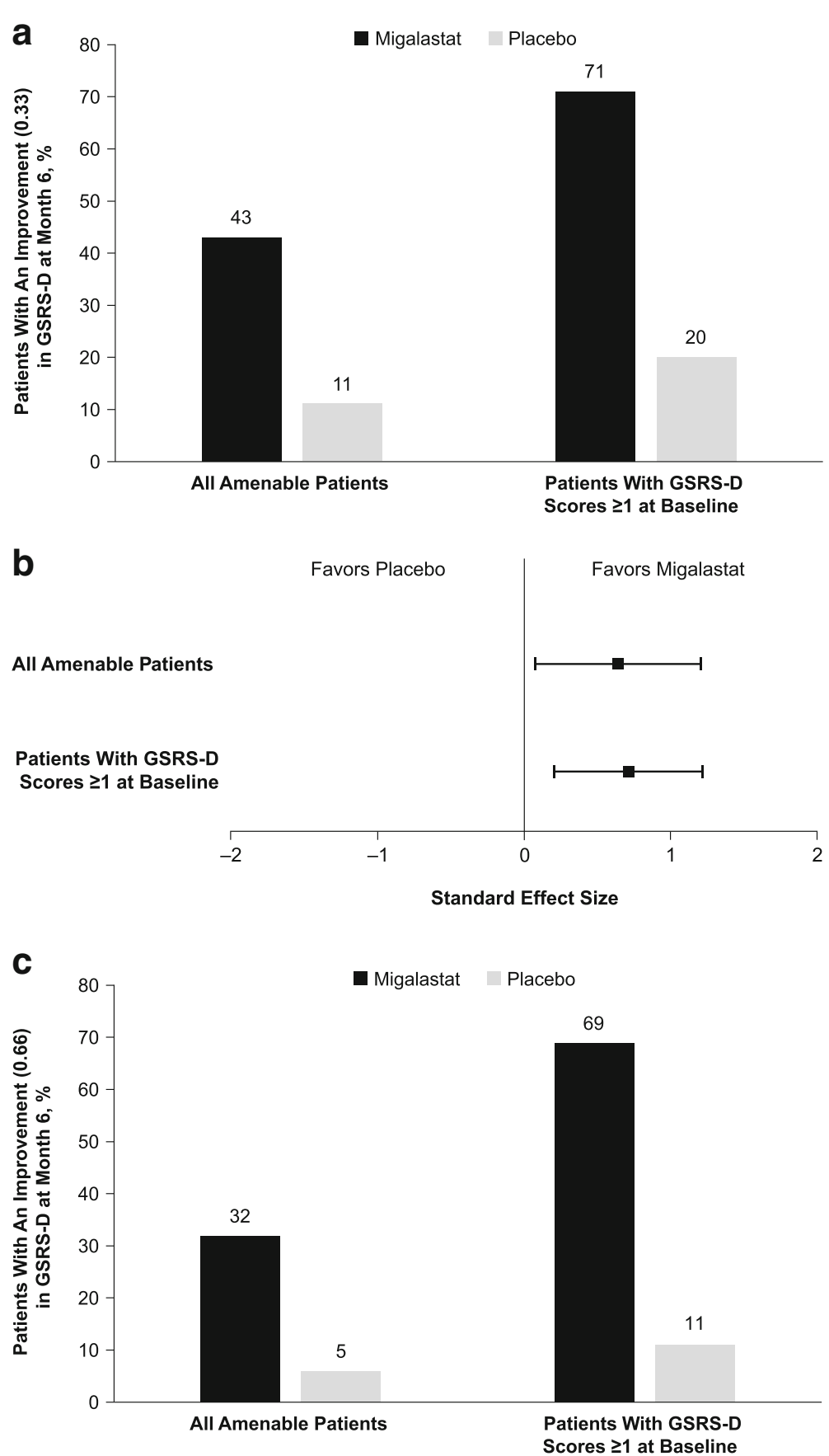

Fig. 1 Patients experiencing a minimal clinically important difference in GSRS-D scores after 6 months of treatment. a Improvement of 0.33. b Forest plot showing the effect size of migalastat treatment vs placebo. c Sensitivity analysis: improvement of 0.66

missing data, the odds ratio was similar for PTC GL3 (odds ratio $=6.6 ; 95 \%$ confidence interval, $1.3-33.0$; $p=.02$ ) and plasma lyso- $\mathrm{Gb}_{3}$ (odds ratio $=6.2 ; 95 \%$ confidence interval, $0.6-64.3 ; p=.12$ ); however, statistical significance was achieved only for PTC GL-3, possibly due to the smaller sample size for plasma lyso-Gb ${ }_{3}$ (PTC GL-3, $n=44$; plasma lyso-Gb $3, n=31$ ).

\section{Discussion}

In this investigation, migalastat was associated with a clinically relevant improvement in diarrhea symptoms based on the GSRS-D in patients with Fabry disease treated during the phase 3 FACETS trial. Based on the estimated MCID of 0.33 , statistically significantly more patients treated with migalastat achieved a clinically 
Table 1 Logistic regression assessing correlation between PTC GL-3 reductions and GSRS-D improvement (patients with amenable mutations)

\begin{tabular}{lll}
\hline Parameter and Criteria & Odds Ratio & $95 \% \mathrm{Cl}$ of Odds Ratio \\
\hline GSRS-D Reduction From & 5.55 & $(1.17-26.26)$ \\
Baseline of $0.33(n=50)$ & & $p=.031$
\end{tabular}

Kidney Peritubular Capillary

GL-3 Reduction From Baseline $>0.1$

Cl confidence interval, GL-3 globotriaosylceramide, GSRS-D Gastrointestinal Symptoms Rating Scale-diarrhea

meaningful improvement in diarrhea symptoms versus those receiving placebo $(43 \%$ vs $11 \% ; p=.02)$ after 6 months of treatment. This statistical significance was maintained when the analysis was restricted to only patients with diarrhea symptoms at baseline $(71 \%$ vs $20 \%$; $p=.02)$. A sensitivity analysis using a higher MCID threshold of 0.66 supports these findings. Additionally, a correlation between reduction of PTC GL-3 inclusions and diarrhea improvement was observed; patients with a PTC GL-3 inclusion reduction of $>0.1$ were 5.6 times more likely to also have improvement in diarrhea symptoms $(p=.031)$. The correlation between reduction in the other disease substrate, plasma lyso- $\mathrm{Gb}_{3}$, and improvement in GSRS-D was nonsignificant; a small sample size may have confounded this result.

Diarrhea is among the most common and most troublesome of the gastrointestinal symptoms experienced by Fabry patients. The abnormal accumulation of GL-3 in neurons of the peripheral nervous system, resulting in altered autonomic function and gastrointestinal disturbances, may contribute to the high prevalence of gastrointestinal symptoms in patients with Fabry disease [26, 27]. Since up to $67 \%$ of patients report that they experience gastrointestinal symptoms, including diarrhea, some or all of the time [12], and patients report as many as 12 bouts of diarrhea per day [28], symptoms can result in significantly reduced quality of life [11]. Thus, reduction in the severity and frequency of diarrhea can be particularly important for patients. Data from open-label studies with ERT have previously reported improvements in gastrointestinal symptoms following treatment with agalsidase alfa [11] or agalsidase beta [10]. After 12 months of ERT, agalsidase alfa reduced the prevalence of diarrhea by $8 \%$ [11]; similarly, following 6-7 months of therapy with agalsidase beta, episodes of diarrhea were reduced, and remained rare or occasional while therapy was maintained ( $\geq 3$ years) [10]. Our results suggest a similar, clinically relevant improvement in diarrhea symptoms with migalastat treatment.

The analyses presented add to previously published data demonstrating that migalastat improves gastrointestinal signs and symptoms in patients with Fabry disease. In the FACETS study, gastrointestinal signs and symptoms were common, with diarrhea occurring in $56 \%$ of patients with amenable mutations at baseline based on the diarrhea domain of the patient-reported GSRS [15]. As previously reported, 6 months of treatment with migalastat resulted in a significant improvement in diarrhea and reflux compared with placebo-treated patients; for diarrhea, this improvement was sustained over 24 months [15]. Over this time, there was also a significant improvement in indigestion and a trend towards improvement in constipation [15].

In the FACETS trial, migalastat reduced substrate levels of GL-3 in patients with Fabry disease [15]. It has been postulated in the literature that GL-3 deposition in endothelial intestinal vasculature and enteric ganglia may contribute to the gastrointestinal manifestations of Fabry disease, with both cell types shown to accumulate GL-3 in Fabry patients with gastrointestinal symptoms $[10,29,30]$. Abnormal function of the enteric plexi is recognized as a potential mechanism causing irritable bowel syndrome, for which patients report similar gastrointestinal symptoms to Fabry disease [28]. Although GL-3 levels in the gastrointestinal tract were not assessed in this study, we hypothesized that other measures of disease substrate (i.e., PTC GL-3 and plasma lyso- $\mathrm{Gb}_{3}$ ) would likely reflect GL-3 changes in the gastrointestinal system, and may be correlated with improvements in gastrointestinal symptoms. Thus, we explored the correlation between reduction in kidney GL-3 inclusions and improvement in GSRS-D scores. The results indicate that reductions in PTC GL-3 inclusions were significantly associated with improvements in diarrhea (GSRS-D scores). Possibly due to the smaller data set available for lyso- $\mathrm{Gb}_{3}$, no statistically significant association was observed between plasma lyso- $\mathrm{Gb}_{3}$ and GSRS-D scores. Additional studies exploring the potential for plasma lyso- $\mathrm{Gb}_{3}$ to be used as a surrogate endpoint are warranted. Based on these results, reductions in GL-3 could be useful as a surrogate endpoint for predicting clinical benefit (i.e., improvement in diarrhea) with migalastat in patients with Fabry disease.

One limitation of these analyses is that the GSRS has not been validated specifically in Fabry disease. Nonetheless, acceptable psychometric properties of the GSRS, including reliability, stability, and construct validity, have been established in patients with irritable bowel syndrome, gastroesophageal reflux disorder, and dyspepsia $[18,31-33]$. Across these studies, the GSRS-D has demonstrated consistently strong psychometric properties with reliability (Cronbach's $\alpha$ ) between 0.72-0.84, test-retest stability (intra-class correlation coefficient) between $0.38-0.70$, and construct validity, as evidenced by correlations with various health-related quality of life instruments including SF-36, Quality of Life in Reflux and Dyspepsia (QOLRAD), and Psychological General 
Well-being (PGWB) [32, 33]. These psychometric properties make a case for further use, examination, and perhaps, validation, of the GSRS in the Fabry patient population. An additional limitation is that the reported $p$-values are nominal $p$-values that have not been adjusted for multiplicity. As such, these should be interpreted with caution.

\section{Conclusions}

To our knowledge, the FACETS study is the only doubleblind, placebo-controlled study to evaluate gastrointestinal signs and symptoms in patients with Fabry disease. Responder analyses demonstrate that migalastat provided a clinically meaningful reduction in diarrhea in patients with Fabry disease and amenable mutations. These data add to the evidence that migalastat improves gastrointestinal signs and symptoms, including diarrhea, reflux, and indigestion, in patients with Fabry disease [15]. Correlations between GSRS-D scores and PTC GL-3 inclusion reductions suggest that PTC GL-3 inclusions are a potential surrogate endpoint that may predict clinical outcomes with migalastat treatment in Fabry disease. Given that diarrhea occurs frequently and is highly troublesome in Fabry disease, patients with an amenable GLA mutation may derive meaningful symptom relief from treatment with migalastat.

\section{Abbreviations \\ ERT: Enzyme replacement therapy; GL-3: Globotriaosylceramide; GSRS: Gastrointestinal Symptom Rating Scale; GSRS-D: Gastrointestinal Symptom Rating Scale-diarrhea; lyso-Gb Gl $_{3}$ Globotriaosylsphingosine; MCID: Minimal clinically important difference; PGWB: Psychological General Well-being; PTC: Kidney peritubular capillary; QOLRAD: Quality of Life in Reflux and Dyspepsia}

\section{Acknowledgements}

The authors acknowledge the scientific writing services of Virginia Owen, PhD. Additional writing and editorial assistance was provided by Sally Mitchell, PhD, and Dana Francis, PhD, from ApotheCom (Yardley, PA); this assistance was funded by Amicus.

\section{Funding}

This study was funded by Amicus Therapeutics, Inc., Cranbury, NJ.

\section{Availability of data and materials}

Not applicable; data are presented in the main paper.

\section{Authors' contributions}

The study (AT1001-011) was designed by the authors and the sponsor (Amicus). Data collection and analyses were undertaken by the sponsor (Amicus) in collaboration with a core group of investigators. The first draft of the manuscript was written by RS and reviewed by all the authors. All authors vouch for the completeness and accuracy of the data and analyses and for the fidelity of the study to the protocol. All authors made the decision to submit the manuscript for publication. All authors read and approved the final manuscript.

\section{Ethics approval and consent to participate}

Written consent to participate in the study was obtained from all patients. The study was approved by the institutional review board or ethics committee at each participating center and was conducted in accordance with the International Conference on Harmonisation Good Clinical Practice guidelines and the principles of the Declaration of Helsinki.

\section{Competing interests}

RS has served as a consultant for and received research funding from Protalix Biotherapeutics and Amicus. DGB has received research funding, serves as a consultant, and is on the speaker's bureau for Amicus and Genzyme, and has received research funding from Shire. AJ has received advisory honoraria and speaker's fees from Shire, Amicus, Biomarin, and Genzyme. DAH has served as a consultant for and received research funding and honoraria from Amicus, Shire, Genzyme, Protalix, and Actelion. RG has received honoraria from Amicus, Biomarin, Genzyme, and Shire. UFR reports other support from Amicus during the conduct of the study, grant support and speaker's honoraria from Amicus, Genzyme, and Shire HGT outside the submitted work, and research funding from Novo Nordisk Research Foundation. SPS reports grants and non-financial support from Amicus during the conduct of the study. LB has served as a consultant for Protalix. RBC has served as a consultant for Amicus and has received grants from the National Institutes of Health. JCJ does not have anything to disclose. KN has served as an advisor for Amicus, Shire HGT, and Genzyme, has received research support from Amicus and Shire HGT, and has received travel support from Genzyme. FH, AM, JPC, NS, and JAB are employees of and hold stock in Amicus.

\section{Publisher's Note}

Springer Nature remains neutral with regard to jurisdictional claims in published maps and institutional affiliations.

\section{Author details}

${ }^{1}$ Baylor Scott \& White Research Institute, Dallas, TX, USA. ${ }^{2}$ Hôpital du Sacré-Coeur, University of Montreal, Montreal, Quebec, Canada. ${ }^{3}$ Salford Royal Foundation Trust, Manchester, Greater Manchester, UK. ${ }^{4} \mathrm{NHS}$ Foundation Trust, Royal Free Hospital, London, UK. ${ }^{5}$ Medical Genetics Service, HCPA/UFRGS, Porto Alegre, Brazil. ${ }^{6}$ Rigshospitalet, University of Copenhagen, Copenhagen, Denmark. 'Emory University School of Medicine, Atlanta, GA, USA. ${ }^{8}$ Miller School of Medicine, University of Miami, Miami, FL, USA. ${ }^{9}$ Massachusetts General Hospital, Harvard Medical School, Boston, MA, USA. ${ }^{10}$ School of Medicine, University of North Carolina at Chapel Hill, Chapel Hill, NC, USA. ${ }^{11}$ Amicus Therapeutics, Inc., Cranbury, NJ, USA. ${ }^{12}$ Department of Nephrology, Royal Melbourne Hospital, Parkville, VIC, Australia. ${ }^{13}$ Present Address: UC Davis MIND Institute, Sacramento, CA, USA. ${ }^{14}$ Institute of Metabolic Disease, 3812 Elm Street, Dallas, TX 75226, USA.

Received: 29 November 2017 Accepted: 18 April 2018

Published online: 27 April 2018

\section{References}

1. Meikle PJ, Hopwood JJ, Clague AE, Carey WF. Prevalence of lysosomal storage disorders. JAMA. 1999;281(3):249-54.

2. Desnick RJ, loannou Y, Eng CM. a-Galactosidase A deficiency: Fabry disease. The online metabolic and molecular bases of inherited disease. 2016; https://doi.org/10.1036/ommbid.181.

3. Germain DP. Fabry disease. Orphanet J Rare Dis. 2010;5:30,

4. Rigoldi M, Concolino D, Morrone A, et al. Intrafamilial phenotypic variability in four families with Anderson-Fabry disease. Clin Genet. 2014;86(3):258-63.

5. Messina S, Tortorella G, Concolino D, et al. Congenital muscular dystrophy with defective alpha-dystroglycan, cerebellar hypoplasia, and epilepsy. Neurology. 2009;73(19):1599-601.

6. Eng CM, Banikazemi M, Gordon RE, et al. A phase 1/2 clinical trial of enzyme replacement in fabry disease: pharmacokinetic, substrate clearance, and safety studies. Am J Hum Genet. 2001;68(3):711-22.

7. Schiffmann R, Murray GJ, Treco D, et al. Infusion of alpha-galactosidase a reduces tissue globotriaosylceramide storage in patients with Fabry disease. Proc Natl Acad Sci U S A. 2000;97(1):365-70.

8. Buda P, Ksiazyk J, Tylki-Szymanska A. Gastroenterological complications of Anderson-Fabry disease. Curr Pharm Des. 2013;19(33):6009-13.

9. Mehta A, Ricci R, Widmer U, et al. Fabry disease defined: baseline clinical manifestations of 366 patients in the Fabry outcome survey. Eur J Clin Investig. 2004;34(3):236-42

10. Banikazemi M, Ullman T, Desnick RJ. Gastrointestinal manifestations of Fabry disease: clinical response to enzyme replacement therapy. Mol Genet Metab. 2005;85(4):255-9. 
11. Hoffmann B, Schwarz M, Mehta A, Keshav S. Gastrointestinal symptoms in 342 patients with Fabry disease: prevalence and response to enzyme replacement therapy. Clin Gastroenterol Hepatol. 2007;5(12):1447-53.

12. Gold KF, Pastores GM, Botteman MF, et al. Quality of life of patients with Fabry disease. Qual Life Res. 2002;11(4):317-27.

13. Benjamin ER, Della Valle MC, Wu X, et al. The validation of pharmacogenetics for the identification of Fabry patients to be treated with migalastat. Genet Med. 2017;19(4):430-8.

14. Galafold [summary of product characteristics]. Buckinghamshire, UK; Amicus Therapeutics UK Ltd: 2017. http://www.ema.europa.eu/docs/en_GB/document_ library/EPAR__Product_Information/human/004059/WC500208434.pdf.

15. Germain DP, Hughes DA, Nicholls K, et al. Treatment of Fabry's disease with the pharmacologic chaperone migalastat. N Engl J Med. 2016;375(6):545-55.

16. Hughes DA, Nicholls K, Shankar SP, et al. Oral pharmacological chaperone migalastat compared with enzyme replacement therapy in Fabry disease: 18-month results from the randomised phase III ATTRACT study. J Med Genet. 2017;54(4):288-96

17. Markham A. Migalastat: first global approval. Drugs. 2016;76(11):1147-52.

18. Svedlund J, Sjodin I, Dotevall G. GSRS — a clinical rating scale for gastrointestinal symptoms in patients with irritable bowel syndrome and peptic ulcer disease. Dig Dis Sci. 1988;33(2):129-34.

19. Barisoni L, Jennette JC, Colvin R, et al. Novel quantitative method to evaluate globotriaosylceramide inclusions in renal peritubular capillaries by virtual microscopy in patients with Fabry disease. Arch Pathol Lab Med. 2012;136(7):816-24.

20. Boutin M, Auray-Blais C. Multiplex tandem mass spectrometry analysis of novel plasma lyso-Gb(3)-related analogues in Fabry disease. Anal Chem. 2014;86(7):3476-83.

21. Benjamin ER, Hamler R, Brignol N, Boyd R, Yu J, Bragat A, et al. Migalastat reduces plasma globotriaosylsphingosine (lyso-Gb3) in Fabry patients: results from the FACETS phase 3 study. J Inherit Metab Dis. 2014;37(Suppl 1):S161.

22. Sterneck M, Settmacher U, Ganten T, et al. Improvement in gastrointestinal and health-related quality of life outcomes after conversion from mycophenolate mofetil to enteric-coated mycophenolate sodium in liver transplant recipients. Transplant Proc. 2014;46(1):234-40.

23. Manger $B$, Hiepe $F$, Schneider $M$, et al. Impact of switching from mycophenolate mofetil to enteric-coated mycophenolate sodium on gastrointestinal side effects in patients with autoimmune disease: a phase III, open-label, single-arm, multicenter study. Clin Exp Gastroenterol. 2015;8:205-13.

24. Chan L, Mulgaonkar S, Walker R, Arns W, Ambuhl P, Schiavelli R. Patientreported gastrointestinal symptom burden and health-related quality of life following conversion from mycophenolate mofetil to enteric-coated mycophenolate sodium. Transplantation. 2006;81(9):1290-7.

25. Xu X, Tian L, Wei LJ. Combining dependent tests for linkage or association across multiple phenotypic traits. Biostatistics. 2003;4(2):223-9.

26. Tuttolomondo A, Pecoraro R, Simonetta I, et al. Neurological complications of Anderson-Fabry disease. Curr Pharmacy Des. 2013;19(33):6014-30.

27. Tuttolomondo A, Pecoraro R, Simonetta I, et al. Anderson-Fabry disease: a multiorgan disease. Curr Pharmacy Des. 2013;19(33):5974-96.

28. Keshav S. Gastrointestinal manifestations of Fabry disease. In: Mehta A, Beck $M$, Sunder-Plassmann $G$, editors. Fabry disease: perspectives from 5 years of FOS. Oxford: Oxford PharmaGenesis; 2006.

29. O'Brien BD, Shnitka TK, McDougall R, et al. Pathophysiologic and ultrastructural basis for intestinal symptoms in Fabry's disease. Gastroenterology. 1982;82(5 Pt 1):957-62.

30. Politei J, Thurberg BL, Wallace E, et al. Gastrointestinal involvement in Fabry disease. So important, yet often neglected. Clin Genet. 2016;89(1):5-9.

31. Wiklund IK, Fullerton S, Hawkey CJ, et al. An irritable bowel syndromespecific symptom questionnaire: development and validation. Scand J Gastroenterol. 2003;38(9):947-54.

32. Kulich KR, Madisch A, Pacini F, et al. Reliability and validity of the gastrointestinal symptom rating scale (GSRS) and quality of life in reflux and dyspepsia (QOLRAD) questionnaire in dyspepsia: a six-country study. Health Qual Life Outcomes. 2008;6:12.

33. Revicki DA, Wood M, Wiklund I, Crawley J. Reliability and validity of the gastrointestinal symptom rating scale in patients with gastroesophageal reflux disease. Qual Life Res. 1998;7(1):75-83.

\section{Ready to submit your research? Choose BMC and benefit from:}

- fast, convenient online submission

- thorough peer review by experienced researchers in your field

- rapid publication on acceptance

- support for research data, including large and complex data types

- gold Open Access which fosters wider collaboration and increased citations

- maximum visibility for your research: over $100 \mathrm{M}$ website views per year

At BMC, research is always in progress.

Learn more biomedcentral.com/submissions 